\title{
LIMITES DE LA NOTION DE RÉCIT MÉDIATIQUE
}

\author{
Noël Nel ${ }^{1}$
}

L'ORM aborde le vaste domaine des productions médiatiques à partir de la notion centrale de récit médiatique. Si elle n'est pas sans pertinence, cette primauté du récit soulève bon nombre de questions.

\section{Valeur et limites de la notion de récit médiatique}

A focaliser sur la dimension narrative des discours médiatiques, on pourrait en arriver vite à ne voir que cette dimension partout. Le danger n'a pas toujours été évité par la narratologie en général, et par l'école greimassienne de Paris en particulier, comme le rappelle Jacques Bres dans son livre sur La narrativité ${ }^{2}$. Le travers est celui de la narrativisation généralisée de toute praxis. A cet égard, les multiples mises en garde de Jean-Michel Adam, qui conseille une conception restreinte du récit médiatique, l'examen attentif des degrés de narrativité, la prise en compte de l'hétérogénéité et de la gradualité des processus, ne peuvent être que les bienvenues.

1 Professeur à l'Université de Metz, fondateur du CREM (Centre de recherche sur les médias) et co-directeur de celui-ci.

2 J. BREs, La narrativité, Louvain-la-Neuve, Duculot, 1994. 
A ce reproche de fascination pour le "tout narratif", que répond l'ORM?

L'affichage de la position de principe est non ambigu : on admet volontiers qu'à côté du récit, il y a des discours et des textes, et on vise une sémiotique de la complexité. Mais les stratégies déployées pour en rendre compte laissent coexister certaines attitudes qui risquent d'enfermer dans une narratologie élargie.

Il s'agit d'abord de ce que l'on pourrait appeler le critère du primat du narratif, notion analogue à ce que André Gaudreault a nommé, dans le domaine du cinéma, la narrativité intrinsèque. Pour André Gaudreault, tout changement de photogramme est narratif. Or Roger Odin a excellemment répliqué qu'il ne faut pas comprendre le récit comme le plus minime changement d'état dans le temps. Et J.-M. Adam a rappelé qu'un récit repose sur une mise en forme narrative, avec nœud et dénouement.

Il faudrait sans aucune doute se méfier aussi du scénario de la contagion exposé par Marc Lits, cette idée que le dispositif médiatique se trouverait contaminé par le modèle narratif, comme l'affirmait déjà Louis Quéré dans les années 1980. Il est bien sûr possible qu'un certain régime de la télévision, celui de la représentation, fiction (série, feuilleton, téléfilm) et information (reportage et magazine) comprises, nécessite le récit. Mais qu'en est-il dans le régime de la présentation et des émissions polylogales ? Et dans celui des émissions de divertissement?

Enfin, si l'on en juge par les contributions précédentes, il faut bien constater l'intérêt de l'ORM pour le récit en général et pas seulement pour le récit médiatique en particulier. Car, à côté des pratiques de médiatisation des membres de l'ORM, on a évoqué les questions de la médiation narrative (le "récit exemplaire" par Jean Verrier, les récits de vie par M. Burgos) et celles de la médiation narrative de pratiques journalistiques de médiatisation. L'ORM seraitil aussi observatoire de la médiation narrative (le récit littéraire) et de la mise en discours de pratiques journalistiques ? Un observatoire du récit total, en quelque sorte?

Sur la question de l'objet de recherche, il pourrait donc paraître judicieux de réévaluer la dimension rhétorique des médias : comment, par exemple, dans leurs "séquences" (au sens de J.-M. Adam) descriptives, explicatives, argumentatives, dialogales, les médias développent-ils une construction rhétorique du discours de la réalité ? Sans doute faudrait-il complexifier la théorie de l'agir médiatique 
sous sa forme télévisuelle : à côté de l'agir téléologique et de l'agir par habitus, incluant tous les deux les stratégies de narration, il faudrait faire leur place à l'agir éthique (valeurs, normes), à l'agir dramaturgique et spectaculaire. Enfin, il faudrait distinguer des degrés et niveaux d'efficacité du récit : micro, meso et macro-récit; récit d'actualité immédiate avec "scénarisation du réel" (Gérard Leblanc) et réactivation de schèmes empruntés aux grandes structurations mythiques. A présent qu'il existe la possibilité de fixer les messages sur supports d'archivage, le travail scientifique peut passer des effets immédiats, puissants ou non, aux effets profonds, et mieux repérer sur les archives la place de la dimension narrative.

\section{Pratique de la démarche en trois étapes}

Suivant explicitement la démarche préconisée par Paul Ricœur, l'ORM tente de reconstituer dans tout processus communicationnel l'articulation des trois mimèsis : préfiguration, configuration, refiguration. Il s'agit là d'une méthode en trois étapes -production, message, réception- propre à l'herméneutique ricœurienne et à l'ensemble de la sémiotique et de la pragmatique contemporaine, dès qu'elle entend se dégager de la clôture structurale. Ainsi, la poétique littéraire de Gérard Genette dépasse à présent le cadre de la seule approche immanente ou interne pour construire le concept de transtextualité, qui révèle la considération attentive des marges, seuils, frontières et indices de passage du texte au hors-texte. Ainsi, l'analyse sémio-discursive de Patrick Charaudeau opte, à propos des médias et tout spécialement de la télévision, pour l'articulation de trois lieux de pertinence (production, discours, réception) et pour la prise en compte d'un interface -une zone qualifiée "interne-externe"au sein de la production et de la réception. On ferait des remarques du même ordre pour la sémio-pragmatique du cinéma que prône Roger Odin. Bref, d'horizons divers se développent des méthodes qui tentent d'articuler de façon rigoureuse texte -transtexte- hors-texte ou contexte. Ces démarches tout à fait nécessaires posent un certain nombre de problèmes que l'on pourrait résumer à trois.

- Quel positionnement pertinent adopter, entre une conception derridienne de l'écriture et du signe comme hiéroglyphe restreint au cœur de la microdiscursivité, et une conception large des logiques 
sociales (Bernard Miège) imposant la primauté de la macrodiscursivité ?

- Quelle théorie de l'agir médiatique, journalistique et télévisuel, retenir, qui articulerait les champs (Pierre Bourdieu) et les lieux de pertinence ( $P$. Charaudeau), pour penser l'économie globale des processus informationnels et communicationnels?

- Comment, enfin, concevoir le paradigme indiciaire permettant de passer des textes et discours aux contextes, sans donner l'impression de faire cohabiter parallèlement des logiques plus ou moins hétérogènes ou incompatibles: logiques de production, logiques de discours (messages, actes, événements), logiques de réception?

Sur ces trois problèmes, le débat a été inégalement productif.

\section{Silence sur l'axe socio-économique de la production}

La question des logiques plus ou moins hétérogènes de la production n'a pas été abordée frontalement, et l'on a focalisé sur les pôles des discours et de la réception. Du coup, la notion d'institution -une des notions majeures de la sphère de production- a été comme une sorte d'impensé. Et, lorsque certaines réalités de la production ont été décrites, par exemple les pratiques journalistiques de type autobiographique, c'est surtout au titre de pratiques individuelles.

\section{Primauté de la dimension narratologique}

L'ORM envisage le récit comme médiateur d'expérience humaine. Il se penche sur les récits de presse, récits d'actualité, récits historiques, récits de l'infra-quotidien, récits de fiction, en mobilisant surtout $P$. Ricœur et des référents théoriques issus de la poétique littéraire (G. Genette), de la sémiotique littéraire (R. Barthes, A. Greimas, U. Eco) et cinématographique (A. Gaudreault). Il serait sans doute nécessaire de mettre au point une approche du récit télévisuel, car ce média oblige à fonder une théorie de l'agir médiatique sous une forme audiovisuelle qui ne soit pas cinématographique; à se pencher sur la dimension technologique de la médiatisation; à modéliser une forme de mise en récit prolongée sur la durée et utilisée comme dispositif hypertextuel ouvert travaillé par le flux.

\section{Difficultés de l'étude de la réception}

Les mises au point de Gérard Derèze et le recours à Marc Augé semblent indiquer que l'ORM entend aborder les processus de réception aux niveaux micro-meso-macrosocial. L'ambition est ample. 
L'ORM parle d'un univers imaginaire attaché aux médias, d'une identité narrative de notre société occidentale médiatisée. Il semble donc postuler une théorie des effets profonds des médias, effets structurant la pensée et l'imaginaire collectifs. En ce sens, il se confronte à des chercheurs comme Paul Virilio (l'infosphère), Régis Debray (la vidéosphère, puis l'infosphère), Pierre Lévy (la pensée collective), ou moi-même (régimes scopiques).

Le travail de Philippe Marion sur la médiagénie semble commencer à aborder ces questions complexes des effets produits par des dispositifs puissants d'information-communication.

Évidemment, ces débats limités ne prétendaient pas aborder la totalité des questions passionnantes que la problématique privilégiée par l'ORM pose à la recherche. Peut-être le temps est-il venu de relayer le souci de cohérence par la quête d'une problématique intégrant la complexité, l'hétérogénéité et la gradualité des processus dans l'analyse des spécificités médiatiques. 